\title{
The Gao beam under a moving inertial load and har- monic compression
}

\author{
Bartłomiej Dyniewicz ${ }^{1, *}$ and Czesław Bajer ${ }^{1, * *}$ \\ ${ }^{1}$ Institute of Fundamental Technological Research, Polish Academy of Sciences
}

\begin{abstract}
In the present work the dynamics of the system of a mass moving on the beam is investigated in detail numerically in the case of vibrations about a buckled state. The differential equation that describes the motion is strongly nonlinear. Simulations are based on the space-time finite element method. It enabled us easily determine the influence of the moving inertial particle. At the computational stage it becomes a real problem when the mass particle traverses joints of neighbouring elements. The results of representative and interesting computer simulations are enclosed.
\end{abstract}

\section{Introduction}

Structural elements that work in nonlinear range behave in a way far from the known for linear ranges. Well known jumps in trusses under finite displacements, natural frequencies varying under axial loads, buckling etc. are good examples. Modern systems can filter entering excitation and respond with selected and prescribed signals. Special structures exhibit non-classical mechanical or rheological properties. These properties can be predetermined to enable developing new metamaterials. Finally special structures that resist to increased loads, reduce the level of vibration or exhibit increased durability. Nonlinear vibration of a compressed beam is here a good example.

The papers devoted to the Gao beam consider the formulation [1-3], analytical and numerical solutions [4-6], contact problems with the Gao beam [7-9] etc. The mathematical analysis of the problem with contact with the proof of the existence of the solution can be found in [10]. Some particular dynamical problems with contact were discussed in $[4,6,8,9]$, where the model, existence of weak solutions, and computer simulations can be found. An interesting problem of the growth of a crack in the Gao beam was studied in [7].

The motion of a point load travelling on a bending element is a typical problem in transportation. Such systems were extensively investigated, however mostly in a classical linear range. In our previous research the noninear Gao beam subjected to a moving massless or inertial load was investigated. The contribution of a nonlinear term together with a traction force exhibited properties unexpected in a linear range.

This paper continues the study of the vibrations of a nonlinear Gao beam that is subjected to a moving mass $[11,12]$ by adding a oscillating axial force. Such

\footnotetext{
*Corresponding author: bdynie@ippt.pan.pl

**Corresponding author: cbajer@ippt.pan.pl
} 
problems arise naturally in transportation systems such as trains or trams. This makes the model, which is a coupled system of the Gao nonlinear beam equation and the motion of the mass, considerably more complex.

In the present work the dynamics of the system of a mass moving on the beam was investigated in detail numerically in the case of vibrations about a buckled state. The simulations are based on the space-time finite element method. It enabled us easily determine the influence of the moving inertial particle. At the computational stage it becomes a real problem when the mass particle traverses joints of neighbouring elements. The simple time integration method separated from the spatial discretization results in complexity in matrix formulation of a resulting systems of algebraic equations (see for example [13]). Moreover, both nonstationary discretization of the doman and nonstationary location of parameters describing moving inertia or granulated stiffness can be applied with this approach relatively easily $[14,15]$. The results of representative and interesting computer simulations are enclosed.

\section{Formulation}

Let us consider the pair of differential equations that govern the motion of the Gao beam (Figure 1)

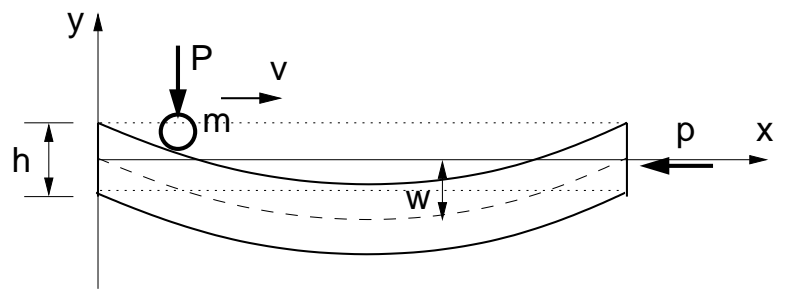

Figure 1. The scheme od the Gao beam.

$$
\begin{aligned}
& \rho A w_{t t}+E I w_{x x x x}-E A(1+\nu)\left[\left((2-\nu) w_{x}^{2} w_{x x}+w_{x x} u_{x}\right]=\left(1-\nu^{2}\right) q(x, t),\right. \\
& \rho A u_{t t}-E A u_{x x}-E A(1+\nu) w_{x} w_{x x}=0 \\
& 0 \leq x \leq L, \quad 0 \leq t
\end{aligned}
$$

with boundary conditions

$$
\begin{aligned}
& w(0, t)=w(L, t)=0, \quad w_{x x}(0, t)=w_{x x}(L, t)=0, \\
& u(0, t)=0, \quad u(L, t)=0 .
\end{aligned}
$$

and initial conditions

$$
w(x, 0)=0, \quad w_{t}(x, 0)=0 \quad \text { and } u(x, 0)=0, \quad u_{t}(x, 0)=0 .
$$

The first equation in (1) describes the bending of the beam, similar to the BernoulliEuler type, with a non-linear term proportional to the axial stiffness and in general to the deflection. The second equation relates to the axial motion. $q(x, t)$ is the distributed external transverse load.

The classical form of the Gao nonlinear beam equation is extended with the coupling of the beam motion with the moving transverse inertial load. The setting is as 
follows. $w(x, t)$ and $u(x, t)$ are transverse and longitudinal displacements, respectively, $\rho$ is the mass density, $A$ - cross section area, $I$ - inertia moment of the cross section, $E$ - Young modulus, and $\nu$ - Poissone coefficient. The lengths of the beam is $L$.

A derivation of the Gao beam model can be found in [1, 16], and related models can be found in the references in the introduction. The beam is clamped at both ends $(x=0, L)$ and a horizontal traction $p$ that may depend on time, acts at the end $x=L$. When the traction is tensile $(p<0)$ the beam behaves essentially as the Euler-Bernoulli beam. However, when the traction is compressive and sufficiently large, say $p>p^{*}>0$, for some threshold $p^{*}$, the beam exhibits two buckled states that are stable and the zero steady state that is unstable. This allows for vibrations about each one of the two buckled states. For the sake of generality, in the formulation we allow the traction $p=p(t)$ to be time dependent and possibly periodic.

We can reduce the system (1) to a single equation. Moreover, the axial vibration can be replaced with constant in length compression, however varying in time. The term $\rho A u_{t t}$ was then put to zero. The given moving mass $m$, the traction $p$, the point load $P$, the mass velocity $\vee$ and the initial data $w_{0}$ and $v_{0}$, allows to write the alternative single equation with the moving mass term in the following form

$$
\begin{gathered}
\rho w_{t t}+k w_{x x x x}-\left(a w_{x}^{2}-\bar{\nu} p\right) w_{x x}=\delta(x-\mathrm{v} t) P-\delta(x-\mathrm{v} t) m w_{t t}(\mathrm{v} t, t), \\
w(0, t)=w_{x}(0, t)=0, \\
w(L, t)=w_{x}(L, t)=0, \\
w(x, 0)=w_{0}(x), \quad w_{t}(x, 0)=v_{0}(x) .
\end{gathered}
$$

Here $\rho$ is the mass density per unit length, $k=2 h^{3} E_{Y} / 3\left(1-\nu^{2}\right), \bar{\nu}=(1+\nu)$, and $a=3 h E, h$ is the heigh of the cross-section, $\nu$ and $E$ are the Poisson ratio and the Young modulus, respectively. $P$ is the vertical point force, $m$ is the inertia of the material point accompanying the force, and $v$ is the velocity of the travelling inertial $\operatorname{load}(P, m)$.

We note here that the vertical acceleration of the mass particle is given by the Renaudot formula [17],

$$
w_{t t}(\mathbf{v} t, t)=\left.\left[w_{t t}+2(\mathbf{v}+\dot{\mathbf{v}} t) w_{x t}+(\mathbf{v}+\dot{\mathbf{v}} t)^{2} w_{x x}+(\ddot{\mathbf{v}} t+2 \dot{\mathbf{v}}) w_{x}\right]\right|_{x=\mathbf{v} t} .
$$

where the derivatives on the right-hand side are evaluated at $x=\mathrm{v} t$, and here and below a dot above a function indicates its time derivative. For the sake of simplicity, we assume that the velocity $v$ is constant, hence, the expression reduces to

$$
w_{t t}(\mathrm{v} t, t)=\left.\left(w_{t t}+2 \mathrm{v} w_{x t}+\mathrm{v}^{2} w_{x x}\right)\right|_{x=\mathrm{v} t} .
$$

The system is nonlinear and so it is natural to consider a weak or variational formulation.

\section{Numerical model}

The differential equation (4) allows us to write the virtual work in the finite element as follows

$$
\Pi=\int_{\Omega} v^{*}\left\{\rho w_{t t}+k w_{x x x x}-a w_{x}^{2} w_{x x}+\bar{\nu} p w_{x x}+\delta(x-\mathrm{v} t) m w_{t t}(\mathrm{v} t, t)-\delta(x-\mathrm{v} t) P\right\} \mathrm{d} x \mathrm{~d} t,
$$


where the virtual velocity $v^{*}$ with the parameter $\alpha$ is given by

$$
v^{*}(x, t)=\delta(t-\alpha h)\left(\mathbf{N v}^{*}\right)^{T} .
$$

We use this form, chosen from the various possible functions of virtual velocity distributions, because of the simplicity of the time integration of the energy functional $\Pi$ and simple stability control. Here, $\alpha \in[0,1]$ is a parameter that defines the equilibrium point in the time layer (similarly to $\beta$ in the Newmark time integration scheme). It affects the accuracy and stability of the resulting time integrating scheme (for details see [18]). Integrating, formally, by parts the virtual work expression (10) leads to the following expression

$$
\begin{aligned}
\Pi= & k \int_{\Omega} v_{x x}^{*} w_{x x} \mathrm{~d} \Omega+\int_{\Omega} v^{*}\left\{\rho \mathbf{v}_{t}-a w_{x}^{2} w_{x x}+\bar{\nu} p w_{x x}\right. \\
& \left.+\left.\delta(x-v t) m\left(v_{t}+2 \mathbf{v} v_{x}+\mathrm{v}^{2} w_{x x}\right)\right|_{x=v t}-\delta(x-v t) P\right\} \mathrm{d} x \mathrm{~d} t .
\end{aligned}
$$

To proceed with the numerical scheme, we need the following matrices: the element mass and stiffness characteristic matrices are given by

$\mathbf{M}=\rho\left[\begin{array}{cccc}\frac{13 b}{35} & \frac{11 b^{2}}{210} & \frac{9 b}{70} & \frac{-13 b}{420} \\ \frac{11 b^{2}}{210} & \frac{b^{3}}{105} & \frac{13 b^{2}}{420} & \frac{-b^{3}}{140} \\ \frac{9 b}{70} & \frac{13 b^{2}}{420} & \frac{13 b}{35} & \frac{-11 b^{2}}{210} \\ \frac{-13 b^{2}}{420} & \frac{-b^{3}}{140} & \frac{-11 b^{2}}{210} & \frac{b^{3}}{105}\end{array}\right]$

and

$$
\mathbf{K}=k\left[\begin{array}{cccc}
\frac{12}{b^{3}} & \frac{6}{b^{2}} & \frac{-12}{b^{3}} & \frac{6}{b^{2}} \\
\frac{6}{b^{2}} & \frac{4}{b} & \frac{-6}{b^{2}} & \frac{2}{b} \\
\frac{-12}{b^{3}} & \frac{-6}{b^{2}} & \frac{12}{b^{3}} & \frac{-6}{b^{2}} \\
\frac{6}{b^{2}} & \frac{2}{b} & \frac{-6}{b^{2}} & \frac{4}{b}
\end{array}\right]+\bar{\nu} p\left[\begin{array}{cccc}
\frac{6}{5 b} & \frac{11}{10} & \frac{-6}{5 b} & \frac{1}{10} \\
\frac{1}{10} & \frac{2 b}{15} & \frac{-1}{10} & \frac{-b}{30} \\
\frac{-6}{5 b} & \frac{-1}{10} & \frac{6}{5 b} & \frac{-11}{10} \\
\frac{1}{10} & \frac{-b}{30} & \frac{-1}{10} & \frac{2 b}{15}
\end{array}\right] \text {. }
$$

To deal with the nonlinearity, we use a term in which the nonlinearity is frozen at the time $t$ so that $\mathbf{w}^{t}$ and $\mathbf{v}^{t}$ are known,

$$
\mathbf{K}_{n}=a \int_{0}^{b} \mathbf{N}^{T}\left[\mathbf{N}_{x} \mathbf{w}^{t}+\left(\alpha-0.5 \alpha^{2}\right) h \mathbf{N}_{x} \mathbf{v}^{t}+0.5 \alpha^{2} h \mathbf{N}_{x} \mathbf{v}^{t+h}\right]^{2} \mathbf{N}_{x x} \mathrm{~d} x .
$$

The explicit matrix form of $\mathbf{K}_{n}$ is provided in the Appendix.

The general matrices representing the moving mass were derived by using linear shape functions as described in [19],

$$
\begin{aligned}
& \mathbf{M}_{m}=m\left[\begin{array}{cccc}
(1-\kappa)^{2} & 0 & \kappa(1-\kappa) & 0 \\
0 & 0 & 0 & 0 \\
\kappa(1-\kappa) & 0 & \kappa^{2} & 0 \\
0 & 0 & 0 & 0
\end{array}\right], \mathbf{C}_{m}=\frac{2 m \mathbf{v}}{b}\left[\begin{array}{cccc}
\kappa-1 & 0 & 1-\kappa & 0 \\
0 & 0 & 0 & 0 \\
-\kappa & 0 & \kappa & 0 \\
0 & 0 & 0 & 0
\end{array}\right], \\
& \mathbf{e}_{m}^{t}=\frac{m \mathbf{v}}{b h}\left[\begin{array}{c}
(1-\kappa)\left(\mathbf{w}_{2}^{i+1}-\mathrm{w}_{2}^{i}-\mathrm{w}_{1}^{i+1}+\mathbf{w}_{1}^{i}\right) \\
0 \\
\kappa\left(\mathbf{w}_{2}^{i+1}-\mathrm{w}_{2}^{i}-\mathrm{w}_{1}^{i+1}+\mathrm{w}_{1}^{i}\right) \\
0
\end{array}\right] .
\end{aligned}
$$


The upper indices $i$ and $i+1$ are the numbers of neighboring spatial elements traversed by the inertial particle. The lower indices 1 and 2 correspond to left and right nodes in each neighbouring element. The differences are equal to zero except the case the mass travels from one element to another during the time interval $\Delta t$. The moving force vector is given as

$$
\mathbf{F}^{t}=P\left[\begin{array}{c}
1-\kappa \\
0 \\
\kappa \\
0
\end{array}\right] \text {, }
$$

where $\kappa \in[0,1]$ is parameter that describes current position of the moving load in finite element. Finally, the minimization of the functional in (12) and the above matrices lead to our numerical scheme, which is of the marching in time type.

Computations were performed according to the algorithm: given $\mathbf{w}^{t}$ and $\mathbf{v}^{t}$, as well as $\mathbf{e}_{m}^{t}$ and $\mathbf{F}^{t}$, find $\mathbf{w}^{t+h}$ and $\mathbf{v}^{t+h}$ by solving the system

$$
\begin{aligned}
& {\left[\mathbf{M} / h+\mathbf{M}_{m} / h+\alpha \mathbf{C}_{m}+0.5 \alpha^{2} h\left(\mathbf{K}-\mathbf{K}_{n}\right)\right] \mathbf{v}^{t+h}} \\
& -\left[\mathbf{M} / h+\mathbf{M}_{m} / h-(1-\alpha) \mathbf{C}_{m}\right. \\
& \left.-\left(\alpha-0.5 \alpha^{2}\right) h\left(\mathbf{K}-\mathbf{K}_{n}\right)\right] \mathbf{v}^{t} \\
& +\left(\mathbf{K}-\mathbf{K}_{n}\right) \mathbf{w}^{t}+\mathbf{e}_{m}^{t}-\mathbf{F}^{t}=0
\end{aligned}
$$

and

$$
\mathbf{w}^{t+h}=\mathbf{w}^{t}+h\left[(1-\alpha) \mathbf{v}^{t}+\alpha \mathbf{v}^{t+h}\right] .
$$

The data set for a steel beam used in the simulations was as follows:

$L=100[\mathrm{~cm}], \beta=0.015, E=2.07\left[\mathrm{~g} / \mathrm{cm} / \mu \mathrm{s}^{2}\right], \nu=0.3, \rho=7.7 \frac{\beta^{2} L^{2}}{\pi}=5.5175[\mathrm{~g} / \mathrm{cm}]$, $A=\beta^{2} L^{2} / \pi, I=\beta^{4} l^{4} / 4 / \pi^{3}, \quad k=0.57 \frac{\beta^{4} L^{4}}{4 \pi^{3}}=0.0233\left[\mathrm{~g} \mathrm{~cm}^{3} / \mu \mathrm{s}^{2}\right], \mathrm{v}=\frac{\pi \sqrt{k / \rho}}{L} \alpha_{v}=$ $0.002 \alpha_{v}[\mathrm{~cm} / \mu \mathrm{s}], m=100 \rho L=55175[\mathrm{~g}], P=m g=5.4071 * 10^{-5}\left[\mathrm{~g} \mathrm{~cm} / \mu \mathrm{s}^{2}\right]$, $p^{*}=0.71\left[\mathrm{~g} \mathrm{~cm} / \mu \mathrm{s}^{2}\right], g=9.8 \cdot 10^{-10}\left[\mathrm{~cm} / \mu \mathrm{s}^{2}\right]$.

\section{Results}

Numerous interesting results can be depicted since the response of the system depends on several parameters, mainly the stiffness and inertia, beam compression $p$, the load velocity, its inertia, and finally the resulting magnitude of deflection. Figure 2 depicts displacements under the inertial load during the passage of a series of loads. The distance between successive inertial loads was equal to the beam length. Four passaged were depicted, each line with increasing compression $p$, constant in time. In the case of zero compression the first stage exhibits significant deflection under the load while in successive stages the inertia of the load stabilizes the trajectory and pulls the beam toward zero deflection. The increased $p$ makes the beam soft and sensitive to the initial stage of vibration. In the extreme cases the beam deflects to the opposite side to the load direction. It is visible in Figure 3. Significant differences between dynamical responses of the beam under constant and oscillating axial compression is depicted in Figure 4. In the case of a moving load the oscillation of $p$ reduces deflection under the load while the influence of a moving inertia increases amplitudes. Differences in both diagrams in Figure 4 are significant and the phenomenon should be investigated further. 


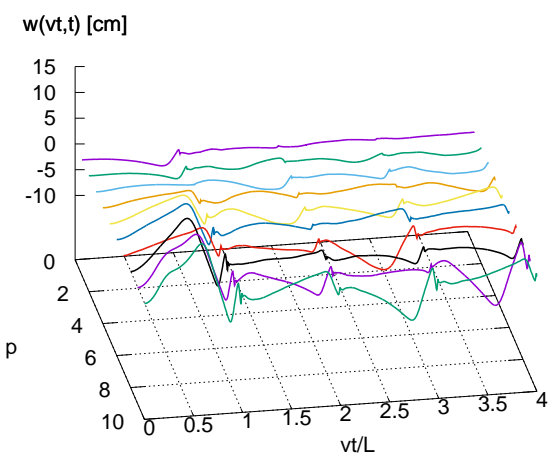

Figure 2. Trajectory of the contact point with the Gao beam subjected to a sequence of inertial forces for increasing $p$.

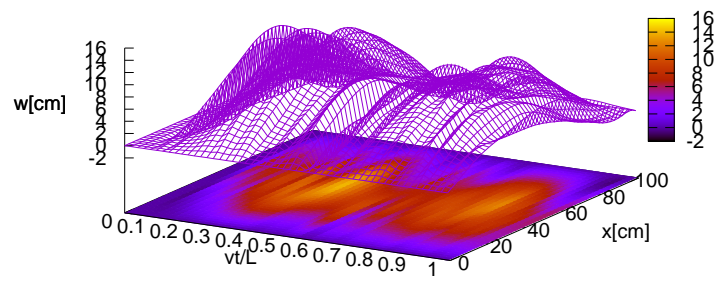

Figure 3. Displacements in time for $\alpha_{v}=0.05, \beta=0.15$ for inertial load with $p=4.5$.

\section{Conclusions}

The compressed beam that works in a strongly nonlinear range and subjected to a moving load exhibits new properties. The deflection depends on several parameters, mainly on the compressive force $p$ and the contribution of the inertial part of the load. The most important feature is the possibility of deflection in opposite direction to the transverse load. It can occur due to the rotational inertia of the part of the beam just subjected to the inertial point load. The strongly compressed beam then jumps out and is held in the upper position.

Acknowledgment This research has been supported within the projects UMO-2015/ 17/B/ST8/03244 and UMO-2017/26/E/ST8/00532 funded by the Polish National Science Centre, which is gratefully acknowledged by the authors. 


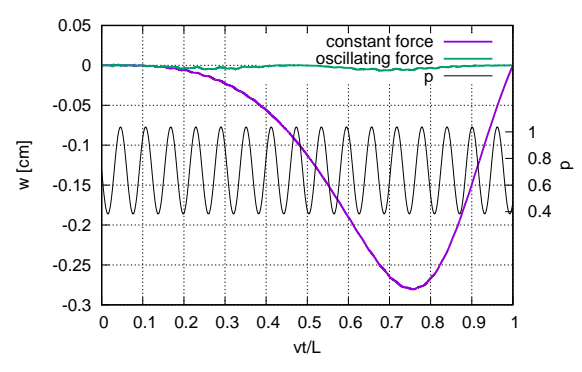

a

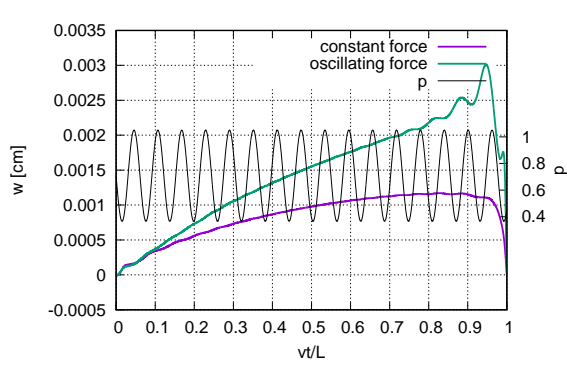

b

Figure 4. Displacements in time of the contact point under the travelling force (a) and the mass (b).

\section{References}

[1] D.Y. Gao. Nonlinear elastic beam theory with application in contact problems and variational approaches. Mechanics Research Communications, 23(1):11-17, 1996

[2] D.Y. Gao and D.L. Russell. An extended beam theory for smart materials applications: II. Static formation problems. Appl. Math. Optim., 38(1):69-94, 1998

[3] D.L. Russell and L.W. White. A nonlinear elastic beam system with inelastic contact constraints. Appl. Math. Optim., 46:291-312, 2002

[4] K.T. Andrews, M.F. M'Bengue, and M. Shillor. Vibrations of a nonlinear dynamic beam between two stops. Discrete and Continuous Dynamical System (DCDS-B), 12(1):23-38, 2009

[5] M.F. M'Bengue and M. Shillor. Regularity result for the problem of vibrations of a nonlinear beam. Electron. J. Diff. Eqns., 27:1-12, 2008

[6] K.T. Andrews, Y. Dumont, M.F. M'Bemgue, J. Purcell, and M. Shillor. Analysis and simulations of a nonlinear dynamic beam. Appl. Math. Physics (ZAMP), 63(6):1005-1019, 2012

[7] K.L. Kuttler, J. Purcell, and M. Shillor. Analysis and simulations of a contact problem for a nonlinear dynamic beam with a crack. Quarterly J. Mech. Appl. Math., 2011 DOI: 10.1093/qjmam/hbr018.

[8] J. Ahn, K.L. Kuttler, and M. Shillor. Dynamic contact of two Gao beams. Electron. J. Diff. Equ., 194:1-42, 2012

[9] K.T. Andrews, K.L. Kuttler, and M. Shillor. Dynamic Gao beam in contact with a reactive or rigid foundation, volume 33, pages 225-248. 2015

[10] M.F. M'Bengue. Analysis of a Nonlinear Dynamic Beam with Material Damage or Contact. PhD thesis, Oakland University, 2008

[11] C.I. Bajer, B. Dyniewicz, and M. Shillor. A Gao beam subjected to a moving inertial point load. Mathematics and Mechanics of Solids, 23(3):461-472, 2018

[12] B. Dyniewicz, C.I. Bajer, K.L. Kuttler, and M. Shillor. Vibrations of a Gao beam subjected to a moving mass (in print). Nonlinear Analysis: Real World Applications, 2019

[13] C.I. Bajer and B. Dyniewicz. Numerical analysis of vibrations of structures under moving inertial load. Springer, 2012 
[14] C. I. Bajer. Adaptive mesh in dynamic problem by the space-time approach. Computers and Structures, 33(2):319-325, 1989

[15] B. Dyniewicz, D. Pisarski, and C. Bajer. Vibrations of a mindlin plate subjected to a pair of inertial loads moving in opposite directions. Journal of Sound and Vibration, 386:265-282, 2017

[16] D.Y. Gao. Finite deformation beam models and triality theory in dynamical post-buckling analysis. Intl. J. Non-Linear Mechanics, 35:103-131, 2000

[17] A. Renaudot. Etude de l'influence des charges en mouvement sur la resistance, des ponts metallique a poutres droites. Annales des Ponts et Chausses, 1:145204, 1861

[18] C. I. Bajer and B. Dyniewicz. Virtual functions of the space-time finite element method in moving mass problems. Computers and Structures, 87:444-455, 2009

[19] B. Dyniewicz. Space-time finite element approach to general description of a moving inertial load. Finite Elem. Anal. and Des., 62:8-17, 2012

\section{A Appendix}

$$
\left.\begin{array}{c}
\mathbf{K}_{n}=\left[\begin{array}{c}
-2\left(b^{2}\left(8 q^{2}-3 q s+s^{2}\right)+3 b(p-r)(3 s-4 q)+12\left(p^{2}-2 p r+r^{2}\right)\right) /\left(35 b^{3}\right) \\
-\left(b^{2}\left(3 q^{2}-2 q s+s^{2}\right)+12 b(p-r)(s-q)+12\left(p^{2}-2 p r+r^{2}\right)\right) /\left(140 b^{2}\right) \\
2\left(b^{2}\left(q^{2}-3 q s+8 s^{2}\right)+3 b(p-r)(3 q-4 s)+12\left(p^{2}-2 p r+r^{2}\right)\right) /\left(35 b^{3}\right) \\
-\left(b^{2}\left(q^{2}-2 q s+3 s^{2}\right)+12 b(p-r)(q-s)+12\left(p^{2}-2 p r+r^{2}\right)\right) /\left(140 b^{2}\right)
\end{array} \mid\right. \\
-\left(b^{2}\left(139 q^{2}-50 q s+25 s^{2}\right)+12 b(p-r)(17 s-13 q)+396\left(p^{2}-2 p r+r^{2}\right)\right) /\left(420 b^{2}\right) \\
-\left(b^{2}\left(2 q^{2}-q s+s^{2}\right)+3 b(p-r)(3 s-q)+18\left(p^{2}-2 p r+r^{2}\right)\right) /(105 b) \\
\left(b^{2}\left(q^{2}+2 q s-53 s^{2}\right)+12 b(p-r)(11 s-q)+108\left(p^{2}-2 p r+r^{2}\right)\right) /\left(420 b^{2}\right) \\
\left.-\left(s^{2}\right)+24 b s(p-r)+36\left(p^{2}-2 p r+r^{2}\right)\right) /(420 b) \\
\quad 2\left(b^{2}\left(8 q^{2}-3 q s+s^{2}\right)+3 b(p-r)(3 s-4 q)+12\left(p^{2}-2 p r+r^{2}\right)\right) /\left(35 b^{3}\right) \\
\left(b^{2}\left(3 q^{2}-2 q s+s^{2}\right)+12 b(p-r)(s-q)+12\left(p^{2}-2 p r+r^{2}\right)\right) /\left(140 b^{2}\right) \\
-2\left(b^{2}\left(q^{2}-3 q s+8 s^{2}\right)+3 b(p-r)(3 q-4 s)+12\left(p^{2}-2 p r+r^{2}\right)\right) /\left(35 b^{3}\right) \\
\left(b^{2}\left(q^{2}-2 q s+3 s^{2}\right)+12 b(p-r)(q-s)+12\left(p^{2}-2 p r+r^{2}\right)\right) /\left(140 b^{2}\right)
\end{array}\right]
$$

where

$$
\begin{gathered}
p=\mathrm{w}_{1}+\left(\alpha-0.5 \alpha^{2}\right) h \mathrm{v}_{1}+0.5 \alpha^{2} h \mathrm{v}_{3}, \\
q=\psi_{1}+\left(\alpha-0.5 \alpha^{2}\right) h \dot{\psi}_{1}+0.5 \alpha^{2} h \dot{\psi}_{3}, \\
r=\mathrm{w}_{2}+\left(\alpha-0.5 \alpha^{2}\right) h \mathrm{v}_{2}+0.5 \alpha^{2} h \mathrm{v}_{4}, \\
s=\psi_{2}+\left(\alpha-0.5 \alpha^{2}\right) h \dot{\psi}_{2}+0.5 \alpha^{2} h \dot{\psi}_{4} .
\end{gathered}
$$

